\title{
A HANDOVER APPROACH TO DVB-H SERVICES
}

\author{
Ville Ollikainen and Chengyuan Peng
}

VTT Technical Research Centre of Finland,
P.O. Box 1000, FI-02044 VTT, Finland

\begin{abstract}
DVB-H offers a new platform for IP-based services and contributes to universal access. There are many challenges of providing multimedia TV experience for DVB-H users anywhere anytime. One big challenge is due to the strict reception conditions and mobility. For example, when a mobile receiver enters a new cell with different transmission frequency, the receiver must accomplish a seamless handover process in order to continue the selected service without an interrupt. Furthermore, it is possible to build DVB-H services into existing DVB-T networks that provide even less possibilities to optimize the transmission for mobile use. This paper presents a novel handover approach to IP streams on a DVB-H network. We handover IP streams by switching between the DVB-H and the UMTS networks without doing any frequency scan. We have successfully tested the service handover approach. The validation has shown that there was no any packet loss during a handover process. The method also provides technology about efficient sharing of wireless network infrastructure with DVB-H, and makes it more feasible to include DVB-H services into existing DVB-T networks and can be used for reducing the time for changing a channel (zapping time).
\end{abstract}

\section{INTRODUCTION TO DVB-H SYSTEM}

Although the terrestrial Digital Video Broadcasting (DVB-T) system has proven its ability to serve fixed, portable and mobile terminals, handheld terminals require specific features from the transmission system. The DVB consortium has agreed on a new standard - the Digital Video Broadcasting for Handheld terminals (DVB-H) for the reliable and efficient delivery of multimedia services over digital terrestrial broadcast networks to mobile handheld devices.

DVB-H is built upon the principles of the DVB-T standard. DVB-T is based on Coded Orthogonal Frequency Division Multiplexing (COFDM) which provides a significant resistance to multi-path distortion and thus also enables the possibility to build Single Frequency Networks (SFN). Existing DVB-T networks, however, have fewer transmitters and larger cell sizes, so SFN is not usually implemented for DVB-H. DVB-H overcomes two key limitations of DVB-T when used for handheld devices [1]. That is it provides means to increase battery usage duration using timeslicing and to improve robustness and improved error resilience using Multi-Protocol Encapsulation-Forward Error Correction (MPE-FEC) in varying transmission conditions and in the mobile reception environments [1]. It also introduced strategies to ease access to the DVB-H services when receivers leave a given transmission frequency - seamless handover as well as optimizing transmitter coverage [2] [3].
DVB-H is a broadcast transmission system based on Internet Protocol (IP) [4]. This allows the DVB-H system to be combined with other IP-based networks. All content in the DVB-H system is carried by IP datagrams and may contain data related to multimedia services, file downloading services, or other services [5]. A general description of the technologies is illustrated in figure 1 [2]. In addition to some DVB-H specific issues, such as conditional access, a full DVB-H system is defined by combining elements in the physical and data link layers as well as service information (PSI/SI) which is shared with the DVB-T system. In physical layer, the following main technical elements were added specifically for DVB-H [1]:

- DVB-H signaling to the DVB-H receivers in the Transmission Parameter Signalling (TPS) bits to enhance and speed up service discovery and possible frequency handover on mobile receivers

- Providing additional $4 \mathrm{~K}$ transmission mode which allows single antenna reception in medium size SFNs at very high physical speed of the receiver

- In-depth symbol interleaver for the $2 \mathrm{~K}$ and $4 \mathrm{~K}$-modes for further improving their robustness in mobile environment and impulse noise conditions

- $\quad$ Enhanced service information [6]

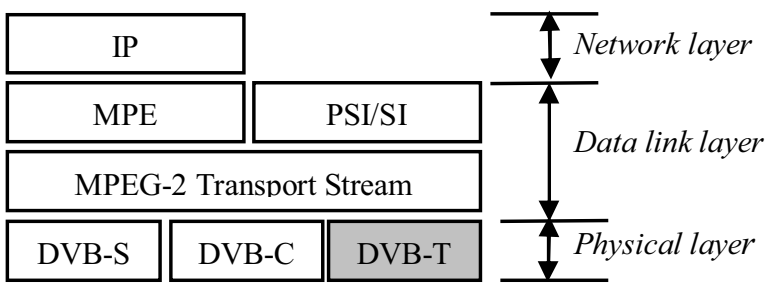

Figure 1: OSI protocol stack-layers 1 - 3 for DVB-H [2]

The IP Datagrams in network layer are encapsulated using MPE in MPE-sections [7]. The key technologies implemented in data link layer are time-slicing and optional MPE-FEC [7] [8]. Handheld devices have small antennas which benefit from receiving signals from many different locations, and also need a robust transmission system with solid error protection. MPE-FEC allows the receiver to improve $\mathrm{C} / \mathrm{N}$ - and Doppler performance in mobile channels and the tolerance to impulse interference [7].

Another technology, time-slicing scheme based on time division multiplexing (TDM), provides a possibility to significantly reduce the average power consumption of a DVB-H terminal [2] [3]. Time-slicing, which is used on elementary streams carrying MPE sections, sends data in bursts at given intervals of time using significantly higher instantaneous bit rate than using traditional streaming mechanisms [2] [7]. In the mean time, the transmission of transport streams is not interrupted while the 
receiver is inactive for periods of time. Between the bursts, data of the elementary stream is not transmitted, allowing other elementary streams to share the bandwidth [2] [7].

Time-slicing also provides a possibility for smooth and seamless service handover by switching the reception from one transport stream to another during an off period [1] [2]. Timeslicing enables a receiver to stay active only a fraction of the time while receiving bursts of a requested service. A major benefit from time slicing to service handover is that the receiver may use the off time to apply a handover strategy that will be discussed in the next section. This period allows the receiver to look for services while the current service is still being displayed.

Despite of these benefits, time-slicing brings in also some drawbacks, especially an increase in zapping time required for changing a channel. It consists of time to tune into a new frequency, to synchronize to the broadcast stream and gathering its metadata, and to wait for the beginning of the next Group of Pictures (GOP) of the elementary stream. Increased zapping time has a negative impact on user experience, and this is why for instance the Nordig consortium has defined a 1.5 second maximum zapping time for ordinary digital television services [14], which is considerably less than a burst interval of over 6 seconds suggested in [2].

\section{HANDOVER STRATEGIES}

A handover by definition is the changes of a communication channel, in this case either a DVB-H or a cellular communications system, while the reception of the currently consumed service is continued [11]. For DVB-H, this implies the change of a transport stream and/or frequency. For the cellular communications system, the change can be for example the change of a network cell.

In a DVB-H network, when a mobile receiver leaves the current reception area, the selected transport stream signal can no longer be received on the tuned frequency. In order to continue the selected service without an interruption, the receiver must accomplish a seamless handover process.

The standard handover strategies suggest that the receiver must first tune to an alternative frequency (i.e. frequency handover), then confirm that the multiplex is carrying the correct service (i.e. service handover), and finally undertake time slice synchronization [2] [3]. Scanning and changing frequencies to find out the exactly same service normally result in a long service interruption that is not acceptable for end users. Accordingly, strategies of seamless handover to handle this situation are essential.

The basic idea of a frequency handover is searching for possible candidate frequencies by signal scanning. Specifically, it scans the whole transmission band or tests specific frequencies to lock to the signal with time slicing indicator transmitted in the TPS-bits in a network [2]. The main method overviewed in the DVB-H standard suggests using the frequency list descriptor in Network Information Table (NIT) [2]. The drawback of this solution is possible tuning failure, although the failure can be improved by other frequency handover strategies such as cell identification via TPS and NIT [2].

After locked to the newly tuned frequency (i.e. undergone frequency handover), a service handover in multiplex level accomplishes switching to the transport stream in this frequency and continues receiving the previously received IP service. A service handover method described in [2] uses IP/MAC Notification Table (INT) tables. The idea is to encapsulate the same IP datagram stream (i.e. same service) in two or multiple transport streams. When handover happens, a receiver may in this way switch to one of the available transport streams to receive the interrupted IP datagram stream. The INT table and its sub-tables need to announce all IP streams both on the actual cell in the current network and on all adjacent cells on other networks [7]. To announce an IP stream, INT contains one or multiple target descriptors, and one or multiple IP/MAC stream location descriptors [7]. Since the amount of frequencies (announced in INT) to be tested is less than other methods, it is a better solution although it may require a relatively large amount of SI information.

Finally, time slice synchronization is essential for seamless handover if the transmitters of two networks have their own DVB$\mathrm{H}$ encapsulators [2]. Since IP network delay and packet jitter may be different for two transmitters of different networks transmitting the same service, if the time slices of the two transmitters are sent out at the same time, they may contain not exactly the same data and therefore cause packet loss when realizing handovers [2]. Two options may overcome this problem. One option is applying a static phase shift between the two networks [2]. Another one is implementing IP encapsulators synchronization [2] by which all transmitted time slice bursts in all the cells have the same content and are transmitted simultaneously. That will be very difficult to implement.

\section{A HANDOVER APPROACH}

We propose a handover method by taking advantage of the Universal Mobile Telecommunications System (UMTS) third generation mobile technology. The main idea is that only one IP datagram stream is encapsulated and sent to the DVB-H network, and the same IP source is sent to a UMTS network. When the tuned signal decreases or the receiver enters a new cell with different transmission frequency, the receiver can then automatically switch to the UMTS network to receive the same service without any interruption. Our approach does not need frequency scanning and synchronization time to tune to the signal of the new cell.

It should be noted that although video broadcast services are planned also for UMTS networks [10], our approach is based on normal unicast data transfer. The UMTS networks are designed for point-to-point transmission, any IP datagram stream can be carried over a unicast IP tunnel. Because the UMTS data service is packet switched, the IP connection can be kept open all the time without significant losses in any resource as long as no data is carried.

\subsection{DVB-H Network Design}

Figure 2 illustrates the simplified DVB-H network architecture in the experiment. In broadcast head-end side, the primary servers include a video/audio streaming server and a DVB-H encapsulator which are placed on the private DVB-T network.

The streaming server sends one single shared IP multicast stream to the DVB-H encapsulator and another unicast stream to a DVB-H client. The DVB-H encapsulator captures the IP multicast packets sent by the streaming server and then encapsulates them in a DVB transport stream. A DVB-H service is then distributed as a single transport stream to the transmitter and is broadcast over-theair to receivers.

The IP multicast or unicast source is the streaming server which gets the content from a MPEG-4 file or live stream encoder 
(cf. figure 2). Video and audio streaming inside the DVB-H encapsulator is based on IP multicast protocol both on IPv4 and IPv6. The streaming server is also configured as a relay source server able to transmit the stream to a relay server.

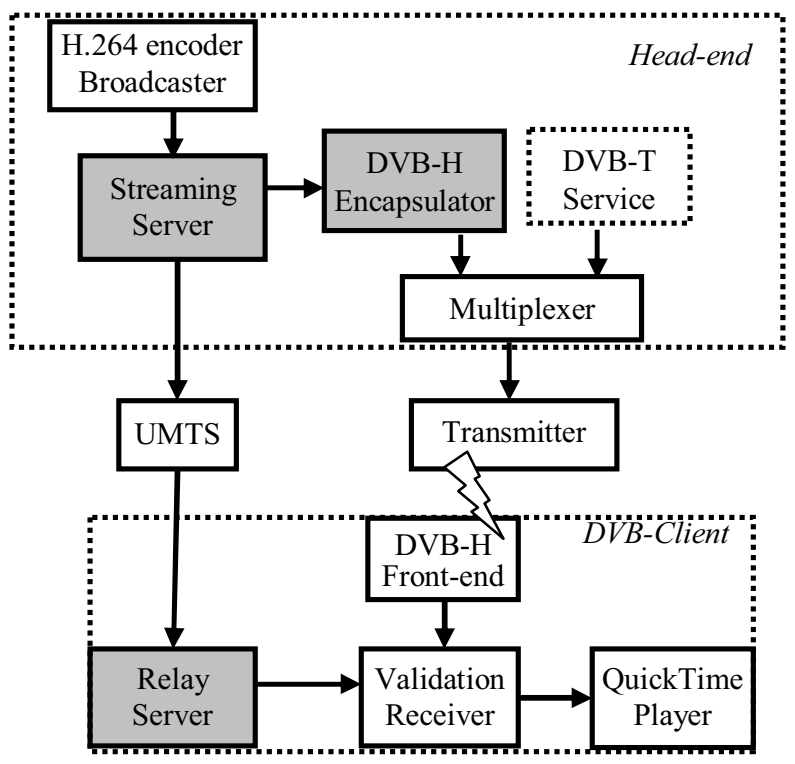

Figure 2. A DVB-H network architecture

In DVB-H client side, the grey colored relay server acts as a relaying streaming server which reflects the received unicast stream again to a shared IP multicast stream to be used by the validation receiver. The validation receiver can then tune in to the local relay when handover happens, thus reducing traffic across the UMTS network.

A DVB-H client checks the antenna signal strength every 10 seconds. When the signal strength has dropped below a preset threshold (e.g. below $30 \%$ of a total strength), the reception of the IP service (i.e. IP datagram stream carrying the same service) can continue on the UMTS network. The switchover criteria could also depend on other criteria, e.g. bit error rates before the final error correction.

\subsection{Implementation}

In the trial implementation we used a laptop as a portable terminal. It was connected to a DVB-H validation receiver with a sample implementation of a DVB-H decapsulator which provides, e.g., buffering of time sliced data, routing of IP packets to a virtual network device, optional measuring of signal strengths, etc.

QuickTime player was selected as a real time application. It receives and playbacks a video/audio stream through reading a Session Description Protocol (SDP) file which contains information from the player to discover, join, and participate in a multimedia session. The multimedia session consists of a set of media streams that exist for some duration of time [9]. The DVB-H encapsulator, the streaming server, the encoder or the broadcaster, the relay client, and other DVB-H clients use the same SDP file so that the correct session parameters are selected during a handover.

During and after handover, a DVB-H client will receive a reflected multicast stream of the unicast stream relayed from the streaming server by reading the IP multicast SDP file. The information given in a SDP file depends strongly on the application used in a terminal because the fields vary a lot. Table 1 lists a sample SDP file used in the application. It includes, e.g., session name, time the session is active, the media comprising the session, information to receive the media (addresses, ports, formats), etc.

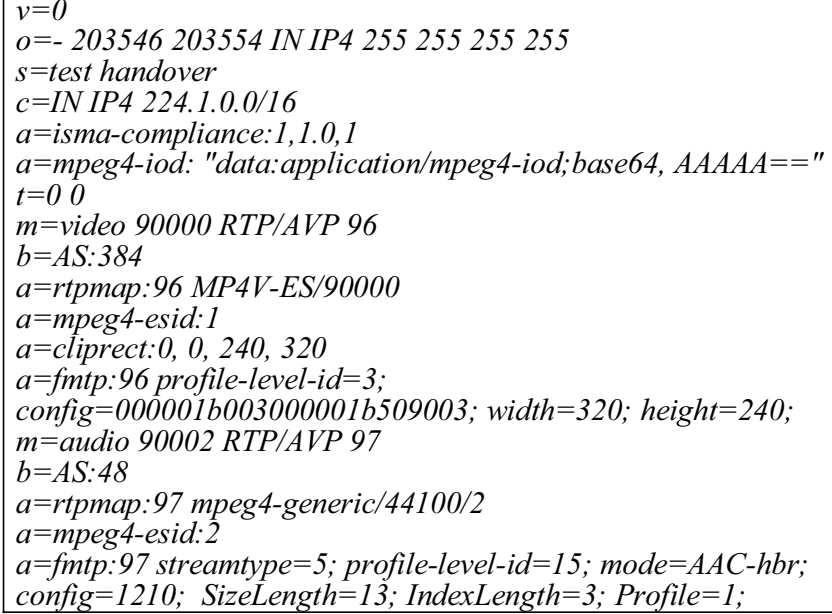

Table 1: An example settings of the SDP file

The DVB-H receiver (cf. figure 2) was comprised of a virtual network adapter and a UMTS mobile connect card. The virtual network adapter was used for routing of UDP packets decapsulated from the DVB-H transport stream which is tuned and received over-the-air. The UMTS mobile connect card was used for reception of UDP packets via UMTS which is a commercial UMTS network run by Elisa Corporation in Finland. The DVB-H also consists of a DVB-H front-end which was used to forward the DVB-H transport stream to the laptop via a USB-port.

The relay server (grey colored box in figure 2) created a Multicast Socket which could listen to a port and to receive UDP Datagram packets destined for the port and the group specified in the SDP file both before and after handover. The MulticastSocket class is provided by java.net package in the Java platform. The socket needs only to join the specified multicast group once, and then it could switch to and back between virtual network and the UMTS network adapters by setting network interfaces.

When the signal strength decreased below the preset threshold, the DVB-H client performed a handover by buffering the received bursts during off-time. That is possible because a mobile handheld terminal requires a constant lower bitrate. The user, however, will not notice the period of receiver activity or inactivity since the data bursts are stored in the receiver memory and played out by the player continuously.

\subsection{Configurations}

The DVB-H network was configured by means of sharing multiplex with our existing Otadigi DVB-T network (cf. figure 2). Thus the transmission mode was set to $8 \mathrm{~K}$ with native symbol interleaver. Time-slicing support was indicated in SI to accept DVB-H signaling instead of TPS signaling via the DVB-T modulator. The reception mode was portable indoor with indoor coverage quality about $60 \%$. 
The DVB-H transport stream bandwidth used for the DVB-H MPE streams was set to $6 \mathrm{Mbps}$. The output bitrate for the multiplexer was set to a value $6748 \mathrm{kbps}$ which was compatible with the modulator's required bitrate and delivered by the DVB-T transmission layer at a constant bitrate. Output transport stream buffer size for Asynchronous Serial Interface (ASI) output card was $4096 \mathrm{kbps}$ in the DVB-H encapsulator. The bitrate for IP service was reserved by multiplexing via the DVB-H encapsulator.

The IP buffer size for incoming IP data in the MPE encapsulator is measured in bursts and was set to three bursts. The burst bitrate equals the ASI output rate $(6748 \mathrm{kbps})$. The elementary stream carried one streaming service at a constant bitrate of $384 \mathrm{kbps}$ which is the limit of the UMTS standard [10].

\section{MEASUREMENTS AND ANALYSIS}

All MPE streams used the same cycle time that was calculated using maximum burst size for a MPE stream with highest bandwidth. If this results in a value is greater than maximum cycle time setting (40.95 s) server will reduce burst size to achieve the defined maximum cycle time [12]. Otherwise each burst of service is sequentially concatenated and the remaining part of the cycle is filled with null transport stream packets [12]. Accordingly, burst duration can be accurately managed and can change from one cycle to the following one [12]. The average burst duration in the experiment measured was about $275 \mathrm{~ms}$.

The receiver was designed to execute signal scan on a regular basis (experimental setting was $10 \mathrm{~s}$ ). With the above settings, the reception of a video service was uninterrupted when testing the handover approach. The video bandwidth of a single MPE stream was delivered in about $384 \mathrm{kbps}$ with a very good quality. The new video compression standards H.264/AVC were used. These standards provided videos with a decreased bandwidth by similar picture quality. The encoding settings were also optimized for playback on QuickTime player. The bitrate of the UMTS network could reach up to as high as $328 \mathrm{kbps}$ (ideal bitrare of UMTS network is $384 \mathrm{kbps}$ ).

Off-time parameter had the impact on handover performance. Off-time length depends on used service and burst bitrates [13]. Increasing off-time can optimize the power saving and manage seamless handover. However, it also increases the service access time. When the off-time was $2400 \mathrm{~ms}$, the average power saving measured was $78 \%$ without affecting handover performance.

Parameters such as burst duration and off-time vary between elementary streams as well as between bursts within an elementary stream. The burst bitrate setting was also crucial for the proposed handover as well as the DVB-H service. In the test the ideal burst bitrate was between 10 to 20 times the constant bitrate of the delivered service.

\section{CONCLUTIONS AND DISCUSSIONS}

In this paper, we presented a novel and reliable handover approach between over-the-air media transmissions on DVB-H and UMTS without doing frequency scanning. In current implementation, the tuning failure and time-slicing synchronization can be effectively avoided. Streaming relays in the UMTS network made the most effective use of limited network resources and segmentation of network traffic instead of loading a single network segment with streaming traffic.

The handover issue was also motivated by DVB-T and DVB$\mathrm{H}$ integration. The DVB-H and fixed DVB-T networks possess different properties. Even within a pure DVB-H network, several bandwidth, coverage, and network cost compromises have to be made. If we make a traditional compromise, we either reduce our bandwidth considerably or have to build an expensive network with more transmitters and gap fillers. Combining the two pure network solutions, mixed networks can be created which take advantage of the properties of both [11].

Our approach by using the UMTS backup gives us still enough bandwidth for DVB-T and provides at least some mobility on DVB-H. There are studies which implicate that although mobile receivers are mobile by nature, the use context is stationary, which makes the optimization even less critical and the UMTS more applicable. Also our DVB-H network implementation becomes an example of the convergence of broadcast and mobile technology.

Our approach proved that when the stream is not available in DVB-H, it can be temporarily received from the UMTS network. Although the benefits of the approach are obvious, the overhead of maintaining a backup UMTS network connection exists. The overhead comes from the relay server which was essential to request IP packets from UMTS network. We also left channel change and zapping times to be examined in subsequent studies.

\section{REFERENCES}

[1] EN 302 304, Digital Video Broadcasting (DVB); Transmission System for Handheld Terminals (DVB-H), ETSI, November 2004.

[2] TR 102 377, Digital Video Broadcasting (DVB); DVB-H Implementation Guidelines, ETSI, November 2005.

[3] TR 101 211, Digital Video Broadcasting (DVB); Guidelines on Implementation and Usage of Service Information (SI), ETSI, May 2004.

[4] DVB A098, Digital Video Broadcasting (DVB); IP Datacast over DVB-H: Architecture, November 2005.

[5] DVB A097, Digital Video Broadcasting (DVB); IP Datacast over DVB-H: Use Cases and Services, November 2005.

[6] EN 300 468, Digital Video Broadcasting (DVB); Specification for Service Information (SI) in DVB Systems, ETSI, November 2004.

[7] EN 301 192, Digital Video Broadcasting (DVB); DVB Specification for Data Broadcasting, ETSI, November 2004.

[8] EN 300 744, Digital Video Broadcasting (DVB); Framing structure, channel coding and modulation for digital terrestrial television, ETSI, November 2004.

[9] IETF RFC 2327, SDP- Session Description Protocol, April 1998.

[10] DVB TM 2466, the Convergence of Broadcast \& Telecommunications Platforms, February 2002.

[11] DVB TM 3095. IPDC in DVB-H: Technical Requirements.

[12] TR 101 202, Digital Video Broadcasting (DVB); Implementation Guidelines for Data Broadcasting, 2003.

[13] TR 102 401, Digital Video Broadcasting (DVB); Transmission to Handheld Terminals (DVB-H); Validation Task Force Report, May 2005.

[14] NorDig, Unified Requirements for Integrated Receiver Decoders for use in cable, terrestrial, satellite and IP-based networks, ver 1.0.2. 